\title{
Ultra-Wideband Technology: Standards, Characteristics, Applications
}

\author{
${ }^{1}$ P.S. Sharma, ${ }^{2}$ Sandeep Vijay, ${ }^{\otimes}{ }^{3}$ M. Shukla \\ ${ }_{1,2} \mathrm{Ph} . \mathrm{D}$. Scholar with UTU, Dehradun and ECE Department, DIT University \\ pss@dituniversity.edu.in \\ ${ }^{3}$ Electronics Engineering Department, Harcourt Butler Technical University \\ manojkrshukla@gmail.com
}

Received: 25th June 2020, Accepted: 14th July 2020, Published: 31st August 2020

\begin{abstract}
The boost in the number of mobile users and automated devices has led to urge of high speed communication with low power consumption devices. Another demand is the uninterrupted communication. This has led to the evolution of a technique referred as Ultra Wideband (UWB). UWB is capable of providing very high data rates and has very low power consumption. This technique relies on carrier less transmission of data in the form of pulses over a wide bandwidth and is mainly suited for short range applications. The short pulses add to the robust performance in the presence of multipath effects. Low spectral power density permits amalgamation of multiple signals and also interception. Due to numerous benefits, UWB finds it usefulness in many areas such as wireless communication, radar imaging, medical and indoor positioning. The paper aims to provide an overview of UWB standards, characteristics and applications.
\end{abstract}

\section{Keywords}

Ultra-wide Band (UWB), Indoor Positioning, Medical, Radar Imaging, Wireless Communication

\section{Introduction}

Internet has become an integral part of human lives. The need of net connection has become a necessity. Nowadays, the public places are installing modems to attract customers in the name of free Wi-Fi. But there are some requisitions such as high data rates, noise free voice communication over data and low power consumption. Most wireless applications work on narrowband signals, which have low data rates. Also, some technologies are not interference free except few. The Wi-Fi devices installed at homes cannot cater to the need of the person who is little far from the device, due to multiple walls between them as the concrete structure poses strong resistance to the flow of signals. Next, in offices there are numerous electronics gadgets that are connected to wireless device and each demanding high data rate which is a problem as the narrowband signals have less data speed. Also increase in number of users mean dividing the spectrum into several bands which further reduces data speed. Moreover, in large warehouses it becomes very difficult to track the position of the workers for efficiency monitoring. Subsequently, in hospitals a patient is loaded with the wires from the medical equipments which monitor their vital statistics. Lastly, voice or cell phones controlled consumer electronics has picked up pace in recent times. For the household equipments to respond in real time a strong internet connection is a mandate. A simple solution to all the above problem lies in a rising technology named as Ultra Wideband (UWB).

UWB is a spread spectrum technique that has gained importance in a very short while. The revolutionary feature of this technique is very high speed and low power consumption making it apt for short range communication [1]. It is also referred as impulse radio UWB (IR-UWB) as it transmits in the radio wave ranges over a large bandwidth, thus utilizing the scant resource efficiently [2]. The data at the UWB transmitter is directly modulated in the form of very short pulses, hence the name impulse. Due to the very small time period, the bandwidth occupied is huge. The UWB has been allotted the unlicensed spectrum $(3.1 \mathrm{GHz}-10.6 \mathrm{GHz})$ for its deployment but as services like GPS, Bluetooth are already exploiting the same band, power of UWB waves has been limited so that it does not causes interference to the existing applications. It has very low power spectral density, even lower than white Gaussian noise as can be seen from the Fig.1. The narrowband and spread spectrum techniques transmit data having high power and so are not affected by wideband signals. 


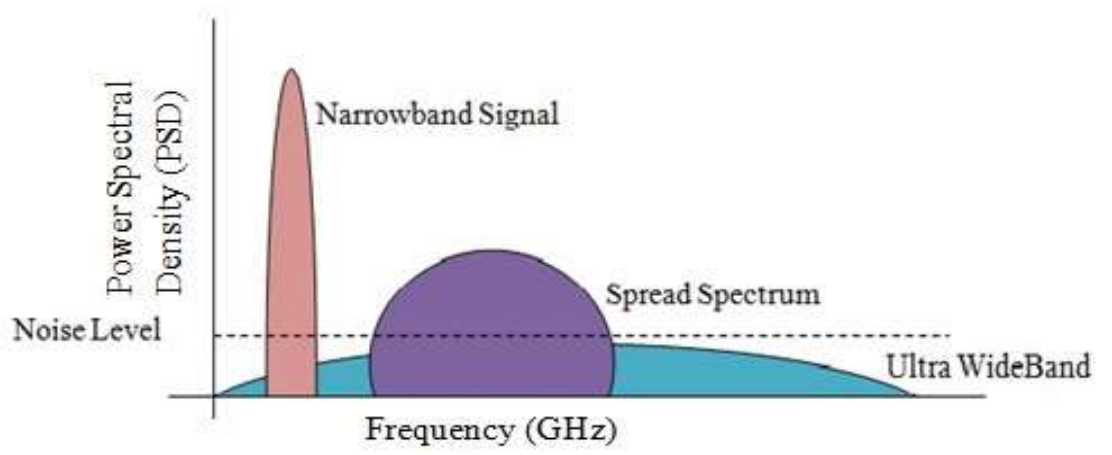

Fig.1: Frequency Spectrum of Ultra WideBand Signal

Ultra wideband is different from other narrowband schemes in terms of signal properties. Firstly, UWB transmits the data on a very wide bandwidth $(\mathrm{GHz})$ as compared to the narrowband transmission employed by other schemes. The order of bandwidth used by UWB is in GHz. Secondly, the other multicarrier techniques either employ frequency, phase or power factors as a carrier for sending of data but UWB transmits data in the form of short pulses of approx 1 ns duration thus making it pulse-position or time dependent technique. Next, in IR-UWB no carrier is required and the data is directly modulated and transmitted over the channel [2]. Lastly, the hardware requirement is very less as compared to other techniques. Power amplifier is a very power hungry device which has no place in UWB transmitter as the signal strength required is very low. Due to omission of carrier, frequency mixer blocks are not essential. Thus, the overall cost is pretty much less.

Ultra wideband technology has several benefits that make it a viable option for multiple applications. First, the short pulses have a small delay spread and the reflection by surrounding objects are dealt by the communication channel itself so it does not pose any problem at the receiver [3,4]. Also, the multipath signals can be combined at the receiver to provide increase in gain. The signal can pass through the walls with limited loss of signal strength thus can provide internet access in every nook and corner of the house. Second, the term ultra-wide signifies that the signal occupies vast bandwidth and thus has very high data rates. In crowded places, multiple users can access the source by employing multiplexing techniques such as code division multiplexing. Next, the short pulse has a large range of sight and thus is beneficial for accurate positioning of targets in large compounds such as a warehouse or an office building [5]. The portable transmitter can be provided to the worker and then movement can be tracked using receivers placed at specific height at suitable locations. Moreover, the exact position calibration feature can provide a solution to monitoring the patient without long wires. A transmitter can be installed above the resting place of the patient and any movement due to respiration can be noticed by the device which can be communicated to the control computer center or sensors can used to read the statistics of the patient. This finds great use in body area networks (BANs) [6]. Additionally, UWB satisfies all the requirements for real time communication in smart homes such as high data speed and long range connectivity [7]. Finally, due to low power spectral density the effect of waves on living beings is negligible which, is a remarkable advantage as research is pointing out the ill-effects of rays from mobile phones, base stations and microwave devices on humans.

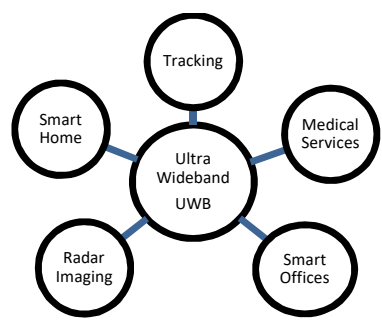

Fig. 2: Applications of Ultra Wideband Technology

The multiple advantages of UWB pave a way for the usage of the technology in various fields such as Smart Homes, Medical Services, Radar Imaging, Smart Offices and Tracking [8] as shown in Fig.2. Each application has a requirement of high data rates, multiple access, low power consuming device and efficient short range communication. The applications are discussed in detail later in the paper. The paper is organized as follows. Section 2 deals with the 
Standards and Regulations defining the specifications and limitations of UWB technology. Next section talks about the characteristics of technique. In Section 4, applications are elaborated. Lastly, the paper is concluded in Section 5.

\section{Standards and Regulations}

Ultra Wideband technology became an area of interest to scientists and researches owing to its multiple advantages but the permit of usage was not sanctioned due to the technical issues such as interference with the already existing GPS and other military applications. But due to continuous appeal, in early 2000's, the Federal Communication Commission (FCC) stated guidelines for the UWB usage [9]. According to the guidelines any signal having bandwidth greater than $500 \mathrm{MHz}$ or having fractional bandwidth more than a quarter of center frequency will be categorized as Ultra Wideband signal [10]. The power spectral density for indoor usage was limited to $-41.3 \mathrm{dBm} / \mathrm{MHz}$, additionally, a slot of $7.5 \mathrm{GHz}$ between the unlicensed band $3.1 \mathrm{GHz}$ and $10.6 \mathrm{GHz}$ was allotted to UWB signal for wireless communication. This was not an issue as UWB has very low power densities in comparison to other narrowband signals using the unlicensed band.

The European Telecommunications Standards Institute (ETSI) followed FCC but with certain stricter conditions [12]. Instead of one single band, two bands were defined by ETSI, a lower band from 3.1-4.8 GHz and upper band from 68.5 GHz. For frequency band 3.4 and $4.8 \mathrm{GHz}$, UWB systems should follow detect and avoid (DAA) principle, which states that the device should look out for any narrowband signals in the band and only if no transmission is detected can the UWB device operate. However, for higher frequency bands DAA can be avoided but power density was limited to $-41.3 \mathrm{dBm} / \mathrm{MHz}$. In 2005, The Ministry of Internal Affairs and Communications (MIC) in Japan also recommended the use of two sub-bands, where the lower band range from 3.4-4.8 GHz and upper band ranges from 7.25-10.25 GHz [13]. DAA condition is necessary for the low band usage. The Electronics and Telecommunications Research Institute (ETRI) reduced the permissible limit of power spectral density in Korea. Further, band allocated for various applications are listed in Table 1.
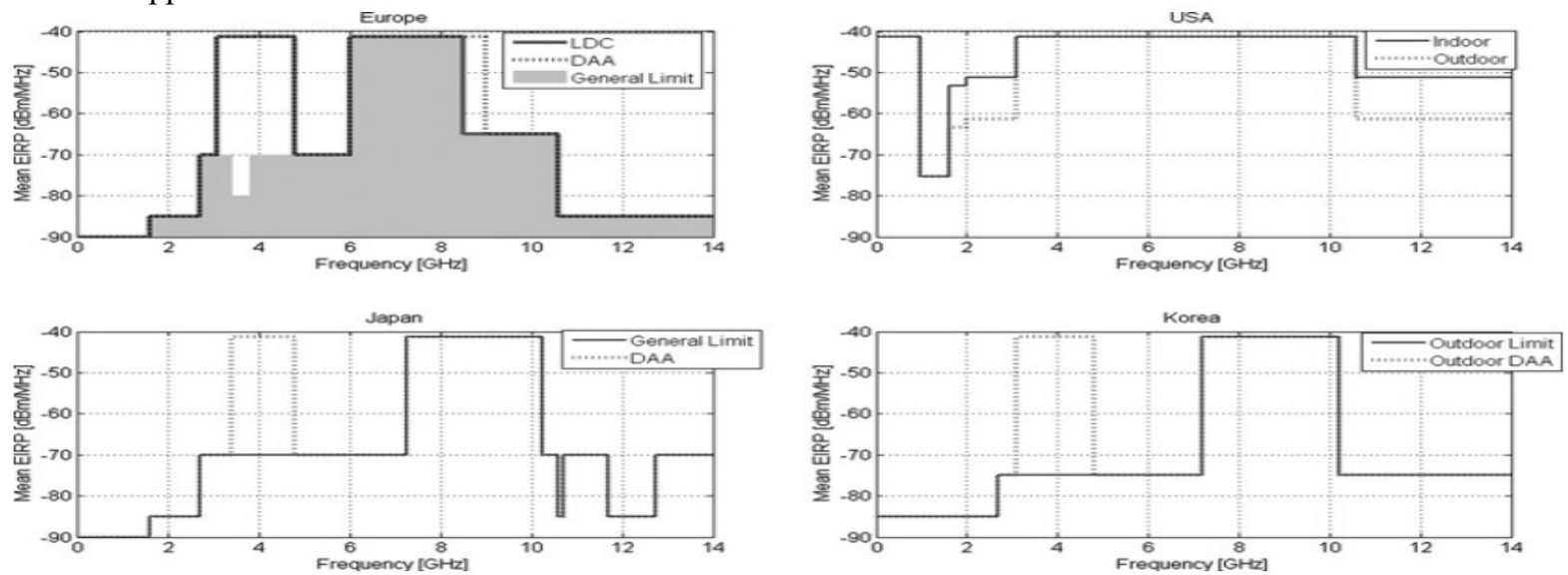

Fig. 3: Ultra Wideband Spectrum Allotment and Limitations [11]

Table 1: FCC Regulations on Ultra Wideband Frequencies

\begin{tabular}{|l|l|l|l|}
\hline S. No & Applications & Bandwidth Allotted & Usage \\
\hline 1 & Communications and Measurement & $3.1-10.6 \mathrm{GHz}$ & $\begin{array}{l}\text { Wireless transmission } \\
\text { and Sensors }\end{array}$ \\
\hline 2 & Vehicular Radar & $24-29 \mathrm{GHz}$ & $\begin{array}{l}\text { Collision avoidance, } \\
\text { Airbag Activation }\end{array}$ \\
\hline 3 & Ground Penetrating Radar & $\begin{array}{l}3.1-10.6 \mathrm{GHz} \text { and below } \\
960 \mathrm{MHz}\end{array}$ & $\begin{array}{l}\text { Research Institutions, } \\
\text { Mining, Detect buried } \\
\text { objects }\end{array}$ \\
\hline 4 & Wall Imaging & $\begin{array}{l}3.1-10.6 \mathrm{GHz} \text { and below } \\
960 \mathrm{MHz}\end{array}$ & $\begin{array}{l}\text { Law Enforcement, } \\
\text { Construction }\end{array}$ \\
\hline 5 & Through Wall Imaging & $\begin{array}{l}1.99-10.6 \mathrm{GHz} \text { and below } \\
960 \mathrm{MHz}\end{array}$ & $\begin{array}{l}\text { Detect Location, Fire } \\
\text { Rescue }\end{array}$ \\
\hline 6 & Medical Imaging & $3.1-10.6 \mathrm{GHz}$ & Medical Personnel \\
\hline 7 & Surveillance & $1.99-10.6 \mathrm{GHz}$ & Public Utilities, Industry \\
\hline
\end{tabular}


The two IEEE standards 802.15.4-2015 and 802.15.6-2012 further define the characteristics of the physical UWB layer in terms of modulation techniques, number of channels, data rate $[14,15]$. The comparison of the two standards in presented in Table 2.

Table 2: Comparison of IEEE Standards

\begin{tabular}{|l|l|l|}
\hline \multicolumn{1}{|c|}{ Parameter } & IEEE 802.15.6-2012 & IEEE 802.15.4-2015 \\
\hline Modulation Technique & $\begin{array}{l}\text { On-Off Keying, } \\
\text { Differential BPSK/QPSK }\end{array}$ & Burst Position Modulation-BPSK \\
\hline Number of Physical Layers & 3 & 18 \\
\hline Ranging & No & Yes \\
\hline Max Data Rate & $15.6 \mathrm{MHz}$ & $27.24 \mathrm{MHz}$ \\
\hline Encoding & BCH & RS, Convolutional \\
\hline Frequency Channels & 11 & 16 \\
\hline Bit Interleaving & Yes & No \\
\hline
\end{tabular}

The standard can be chosen according to the application and the requirement of the user.

\section{Characteristics of UWB}

Ultra Wideband technology is empowered with wide bandwidth, high data rates and low power consumption. Some of the main characteristics of UWB making it a favorable option for wireless communication are discussed in this section.

a) Large Channel Capacity

According to FCC, any signal having bandwidth greater than $500 \mathrm{MHz}$ or has fractional bandwidth greater than a quarter of center frequency is called as Ultra Wideband signal. Wide bandwidth of signal results in greater channel capacity as the capacity of channel is directly proportional of bandwidth. Channel capacity can be defined as the number of bits transmitted per second over the channel. The capacity of UWB can be given calculated from Shannon's Theorem as

$$
C=B \log _{2}\left(1+\frac{\int P(f) d f}{\int N_{o} d f}\right)
$$

Where, C denotes Channel Capacity, B is the Bandwidth, P is Signal Power and N signifies Noise Power. Thus with increase in capacity, data rates of the order of gigabits per second (Gbps) can be attained but due to limitation imposed by FCC only short range communications can avail the advantage. The high data rates assists in real time transfer of data signals, key aspect for the successful operation of smart homes. With high bandwidth, the dependency of capacity on SNR is less and hence UWB signals can survive even in noisy environments.

\section{b) Carrier free Signal and Low Cost}

Unlike most conventional schemes, IR-UWB directly modulates the data in the form of pulses for transmission. This results in simple hardware requirement as many complex devices such as shaping filters, equalizers, digital to analog converters, and frequency mixers are not required and thus accounts for low cost UWB systems. As UWB is employed for indoor applications, power amplifier, which is a very power hungry device, is also not required at the transmitter thus providing additional savings in cost and power consumption. The elimination of many hardware blocks also reduces power requirement. Another benefit of low power is resistant to interception and detection. The eaves dropper has to be in the vicinity for successful reception.

\section{c) Modulation Techniques}

As UWB shares its spectrum with other narrowband and radio signals, to create negligible interference to the existing devices modulation plays a major role. The data in UWB method undergoes pulse shaping in the first step. Pulse Position Modulation (PPM) is employed for shifting of the pulses in time domain resulting in pulses of very small time duration approx 1ns. The nanosecond pulses are impossible to detect as their time of arrival is difficult to predict and thus is very useful in military for communications. However, some PPM sample may not carry any data and cause interference. This can be diminished by spreading and randomizing the signal using pseudo-noise sequences. The randomization of the signal is termed as Time Hopping (TH) [16]. Another way to reduce interference is to increase the time period of the pulses. To enable multiuser transmissions, code division multiplexing is preferred as compared to frequency division as the latter dissects the bandwidth available. Code division multiplexing segregates the user based on the different coding patterns, enabling users to have all bandwidth available. CDMA also prevents the interception of signal by nearby user as the codes are only known to the transmitter and receiver. Furthermore, Orthogonal Frequency Division Multiplexing (OFDM) can be incorporated to avoid interference. 


\section{d) Resistant to Multipath Fading and Jamming}

The multipath fading is very severe in narrowband signals due to the destructive interference of Line of Sight (LOS) and Non-Line of Sight (NLOS) signal. In an indoor atmosphere, many appliances or objects reflect the UWB signal in all possible directions, thus multi-path fading is also an important factor in the performance of UWB devices. The signals while traversing face delay spread but due to short pulse width, spreading is limited resulting in large coherence bandwidth. So possibility of reaching of various components during the same time interval is very faint. Thus, UWB signals are impassive to multipath fading as it posses large bandwidth. Additionally, the multipath components can be combined at the receiver to increase the diversity gain. The UWB waves can also pass through walls as the lower frequencies in the spectrum have large wavelength, hence can penetrate through variety of materials. Another important concept is jamming of signals. Processing gain (PG) is a parameter used to define the jamming capability. It is defined as the ratio of total bandwidth available to the bandwidth used by signal. As the jamming devices are tuned to certain set of frequencies, UWB signals are never completely blocked as compared to narrowband signals.

\section{Applications \\ Ultra Wideband is synonymous to carrier less, short pulse, high data rates and spread spectrum transmission. These features enable multiple user signals and applications to run simultaneously over a given bandwidth. Many systems integrate UWB technology to enhance the performance. The results further stimulate the research for its applicability in other domains. Some of the main applications are elaborated below:}

\section{a) Radar Imaging and Tracking}

Radar Imaging is a technique used to obtain images of objects falling in line of sight (LOS) of the signal. It finds application in detection of wall, in vehicles, underground objects, mining and surveillance. Underground imaging also finds its application in military for assessing the positions of landmines. Tracking deals with the identification of a person or an object inside any boundary or in the vicinity. The short pulses in UWB assist in accurate distance and position calculation with very good resolution which is a prime requirement for Radar systems. The wideband property helps in differentiating between close kept objects and is immune to medium particles and interference. Vehicular radar systems employ UWB signals with directional antenna to detect the presence of moving object, aiding parking and avoidance of collision. In [17], author has presented a thorough survey of UWB and has also explained the position estimation techniques such as angle of arrival, signal strength and time of arrival. In [18], author has implemented the position system and calculated the accuracy of ranging which is reported to be less than $10 \mathrm{~cm}$ for indoor and outdoor environments. The author in [19], studied the effect of power variation on the ranging applications and results show improved performance.

\section{b) Communication}

The dependency on internet has led to the installment of Wi-Fi devices in most public places, offices, industries and houses. The attractive feature of UWB technology is that it transmits over the radio frequency bandwidth and unlicensed band. The vast frequency range facilitates in providing high data rates over short distances. The operation over low frequencies contributes to low attenuation and path loss. UWB signals can also be transported over wires and cables [20]. The low power signals do not pose any resistance in the path of television, voice, data signals already flowing through the wires and cables. In literature, various modulation schemes have been implemented to study and improve the performance of UWB systems. OFDM technique has been applied for improved performance of UWB in [21]. In [22], single carrier UWB (SC-UWB) has been implemented with frequency domain equalization (FDE). In [23], author tried to calculate the bandwidth which can be least affected by multipath components in indoor environment and came to conclusion that a bandwidth of $200 \mathrm{MHz}$ is suitable for immunity to multipath fading. The author in [24], studied the effect of multi-tone frequency shift keying on Ultra wideband signals. Research has also been focused on suitable antenna for Ultra Wideband applications in [25, 26].

\section{c) Medical Field}

The ultra wideband technology is a promising candidate in medical field for communication due to its low signal strength. The low power of the signal is unaffected by the interference in the vicinity and jamming devices and also has a very less impact on human body. For monitoring patient statistics, on-body and in-body sensors are implanted which communicate to nearby control computer centre. The on-body sensor usually communicates about the saturation level, heart rate, temperature and blood pressure of the patient. The sensors free the patients from the wires of the medical instruments. In [27], the author has compared the performance of wireless body area networks (WBANs) in the presence of UWB devices and other wireless systems. In [28], the actual hospital dimensions were utilized to model the channel and simulations were performed taking into account different receiver such as Rake, Energy Detection receivers. 


\section{Conclusion}

Ultra Wideband (UWB) technique relies on carrier-less transmission and has benefits such as high data rates and multiple access. In the paper an overview of the standards applicable to UWB technology in US, Europe and Korea has been discussed. Also IEEE standards 802.15.4-2015 and 802.15.6-2012 has been compared based on modulation techniques, interleaving, coding techniques and number of physical channels. A detailed information on the characteristics of UWB technology has been layout. Additionally, some applications such as radar imaging, wireless communication and medical services have also been dealt with. Inspite of having multiple benefits, the practical implementation of UWB technique is not straight forward. Achieving giga bits per second speed is yet in theory only. Also the signal is affected somewhat by multipath fading and poses some interference to other techniques. The antenna design for UWB transmissions is yet to achieve perfection. These are some areas in which future research can be focused upon.

\section{References}

[1]. Taylor, James D., ed. Ultra-wideband radar technology. CRC press, 2018.

[2]. Win, Moe Z., and Robert A. Scholtz. "Impulse radio: How it works." IEEE Communications letters 2.2 (1998): 36-38.

[3]. Koshelev, Vladimir I., Victor P. Belichenko, and Yury I. Buyanov. Ultrawideband short-pulse radio systems. Artech House, 2017.

[4]. Molisch, Andreas F. "Ultra-wideband communications: An overview." URSI Radio Science Bulletin 2009.329 (2009): 31-42.

[5]. Alarifi, Abdulrahman, et al. "Ultra wideband indoor positioning technologies: Analysis and recent advances." Sensors 16.5 (2016): 707.

[6]. Chavez-Santiago, Raul, Ilangko Balasingham, and Jacob Bergsland. "Ultrawideband technology in medicine: A survey." Journal of Electrical and Computer Engineering 2012 (2012).

[7]. Minoli, Daniel, and Benedict Occhiogrosso. "Ultrawideband (UWB) technology for smart cities IoT applications." 2018 IEEE International Smart Cities Conference (ISC2). IEEE, 2018.

[8]. Rahayu, Yusnita, et al. "Ultra wideband technology and its applications." 2008 5th IFIP International Conference on Wireless and Optical Communications Networks (WOCN'08). IEEE, 2008.

[9]. Niemelä, Ville, et al. "An ultra wideband survey: Global regulations and impulse radio research based on standards." IEEE Communications Surveys \& Tutorials 19.2 (2016): 874-890.

[10]. Federal Communications Commission, "Revision of Part 15 of the Commission's Systems," First Report and Order, ET Docket 98-153, FCC 2002. http://www.fcc.gov 02-48, April

[11]. Sipal, Vit, et al. "Twenty years of ultrawideband: Opportunities and challenges." IET communications 6.10 (2012): 1147-1162.

[12]. European Telecommunications Standards Institute (ETSI), Technical Report, "Technical Characteristics for Short range Devices" Communication Equipment using Ultra-Wideband Technology," TR 101 994-1, V1.0.0, August 2003

[13]. Kohno, Ryuji, and Kenichi Takizawa. "Overview of research and development activities in NICT UWB consortium." 2005 IEEE International Conference on Ultra-Wideband. IEEE, 2005.

[14]. Gutierrez, Jose A., Edgar H. Callaway, and Raymond L. Barrett. Low-rate wireless personal area networks: enabling wireless sensors with IEEE 802.15. 4. IEEE Standards Association, 2004.

[15]. Chavez-Santiago, Raul, et al. "Propagation models for IEEE 802.15. 6 standardization of implant communication in body area networks." IEEE Communications Magazine 51.8 (2013): 80-87.

[16]. Win, Moe Z., and Robert A. Scholtz. "Ultra-wide bandwidth time-hopping spread-spectrum impulse radio for wireless multiple-access communications." IEEE Transactions on communications 48.4 (2000): 679-689.

[17.] Gezici, Sinan, and H. Vincent Poor. "Position estimation via ultra-wide-band signals." Proceedings of the IEEE 97.2 (2009): 386-403.

[18]. Sahinoglu, Zafer. "Improving range accuracy of IEEE 802.15. 4a radios in the presence of clock frequency offsets." IEEE Communications Letters 15.2 (2010): 244-246.

[19]. Ye, Tingcong, Michael Walsh, and Brendan O'Flynn. "IEEE 802.15. 4a UWB-IR ranging with bilateral transmitter power control methodology for multipath effects mitigation." (2013): 37-37.

[20]. Rahayu, Yusnita, et al. "Ultra wideband technology and its applications." 2008 5th IFIP International Conference on Wireless and Optical Communications Networks (WOCN'08). IEEE, 2008.

[21]. Lee, Jeesung, and Hanho Lee. "A high-speed two-parallel radix-24 FFT/IFFT processor for MB-OFDM UWB systems." IEICE Transactions on Fundamentals of Electronics, Communications and Computer Sciences 91.4 (2008): 1206-1211. 
[22]. Wang, Yue, et al. "Cyclic prefixed single carrier transmission in ultra-wideband communications." IEEE Transactions on Wireless communications 5.8 (2006): 2017-2021.

[23]. Sipal, Vit, et al. "Frequency-selective fading of ultra wide band wireless channels in confined environments." IET microwaves, antennas \& propagation 5.11 (2011): 1328-1335.

[24]. Sipal, Vit, Ben Allen, and David Edwards. "Multi-tone frequency shift keying for ultra wide band wireless communications." IET communications 6.10 (2012): 1170-1178.

[25]. Vishal Shukla, Manoj Kumar Shukla and Tanuja Pande. "Multiuser Detection using IDMA Scheme in UWB Home Environment." International Journal of Computer Applications (IJCA), 55.13(2012): 24-29.

[26]. Li, Wen Tao, Xiao Wei Shi, and Yong Qiang Hei. "Novel planar UWB monopole antenna with triple bandnotched characteristics." IEEE Antennas and Wireless Propagation Letters 8 (2009): 1094-1098.

[27]. P.S. Sharma, Sandeep Vijay, M. Shukla. "UWB Based IDMA System with RAKE Reception." Wulfenia: Mitteilungen des Kärntner, 22.4 (2015), 168-183.

[28]. Niemelä, Ville, Matti Hämäläinen, and Jari Iinatti. "IEEE 802.15. 4a UWB Receivers in medical applications." International Journal of Ultra Wideband Communications and Systems 2.2 (2011): 73-82. 